\title{
Predicting synergism of anti-HIV drug pair combinations
}

\author{
Alexey A. Lagunin $^{1,2^{*}}$, Sergey M. Ivanov ${ }^{1,2}$, Roman Yu. Shobik2, \\ Alexandra S. Khromova², Anton S. Smirnov², Nadezhda Yu. Bizyukova², \\ Dmitry A. Filimonov', Olga A. Tarasova', Vladimir V. Poroikov. \\ alexey.lagunin@ibmc.msk.ru
}

(1) Department for Bioinformatics, Institute of Biomedical Chemistry, Moscow, Russia

(2) Department for Bioinformatics, Pirogov Russian National Research Medical University, Moscow, Russia 


\section{Background}

\section{Summary of the global HIV epidemic (2018)}

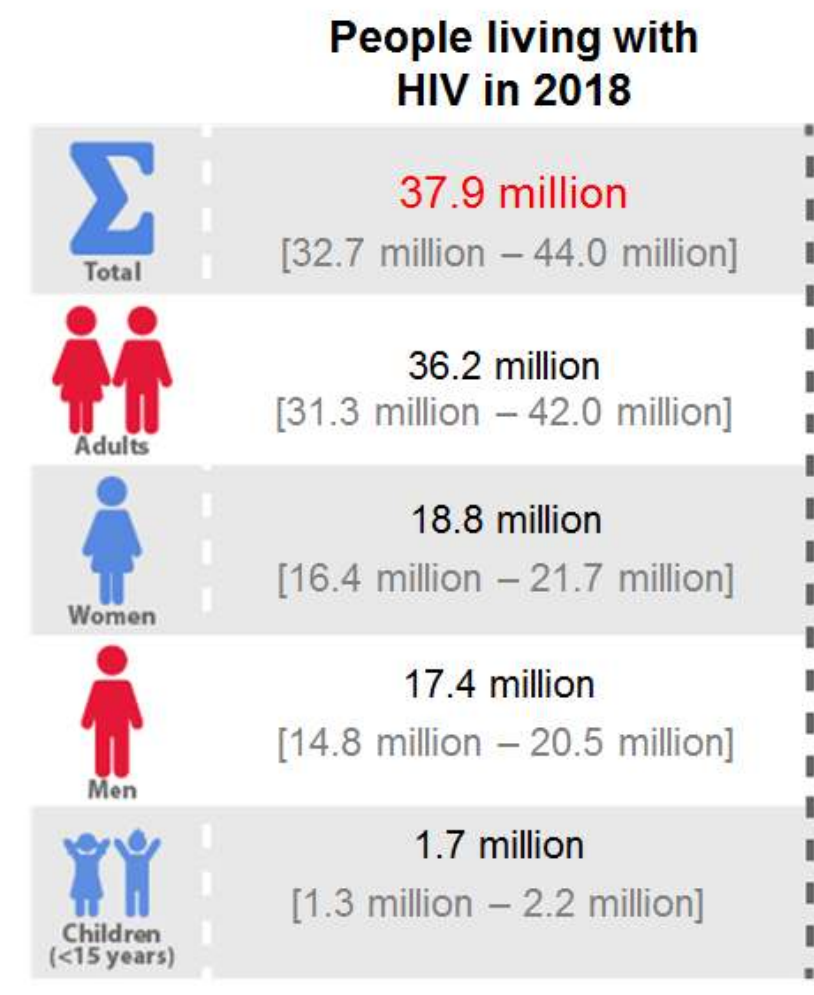

People newly infected with HIV in 2018

1.7 million

[1.4 million -2.3 million]

1.6 million

[1.2 million - 2.1 million]

160000

[110 $000-260000]$
HIV-related deaths 2018

770000

[570 $000-1.1$ million]

670000

[500 $000-920$ 000]

100000

[64 $000-160000]$ 


\section{Antiretroviral drug combinations for the treatment of HIV/AIDS}

- Antiretroviral therapy (ART) including 2 or 3 antiretroviral drugs simultaneously has been successfully used for over 20 years.

- Simultaneous action on different targets decrease possibility to get drug resistance.

- Some combinations of currently used ART may have side effects, toxicity, especially among people living with HIV within the group of patients over 50 years old.

- The number of pairwise combinations for approved drugs is extremely high, therefore we need a rational approach for predicting the synergism of anti-HIV drug pair combinations. 


\section{Experimental data}

500,000 from 1000 FDAapproved drugs or clinically tested drugs and identified drugs that synergize to inhibit HIV replication.

To reduce the number of experiments. The study was made on two stages. It was called MuSIC screen. nature

biotechnology

Systematic identification of synergistic drug pairs targeting HIV

Xu Tan ${ }^{1}$, Long Hu${ }^{2,4}$, Lovelace J Luquette III ${ }^{3,4}$, Geng Gao ${ }^{1}$, Yifang Liu ${ }^{2}$, Hongjing Qu ${ }^{1}$, Ruibin $\mathrm{Xi}^{3}$, Zhi John Lu${ }^{2}$, Peter J Park ${ }^{3}$ \& Stephen J Elledge ${ }^{1}$

The systematic identification of effective drug combinations has been hindered by the unavailability of methods that can explore the large combinatorial search space of drug interactions. Here we present multiplex screening for interacting compounds (MuSIC), which expedites the comprehensive assessment of pairwise compound interactions. We examined $\sim 500,000$ drug pairs from 1,000 US Food and Drug Administration (FDA)-approved or clinically tested drugs and identified drugs that synergize pairs from 1,000 US Food and Drug Administration (FDA)-approved or clinically tested drugs and identified drugs that synerg
to inhibit HIV replication. Our analysis reveals an enrichment of anti-inflammatory drugs in drug combinations that synergize against HIV. As inflammation accompanies HIV infection, these findings indicate that inhibiting inflammation could curb HIV propagation. Multiple drug pairs identified in this study, including various glucocorticoids and nitazoxanide (NTZ), synergize by targeting different steps in the HIV life cycle. MuSIC can be applied to a wide variety of disease-relevant screens to facilitate efficient identification of compound combinations.

HIV has plagued humans for at least 30 years, infecting 60 million tively treated with highly active antiretroviral therapy (HAART), which usually comprises a combination of three anti-HIV drugs ${ }^{1}$. However, the cost of current HAART is prohibitive in developing However, the cost of current HAART is prohibitive in developing countries. In addition, long-term HAAR'T can have serious side effects such as lipodystrophy, hyperglycemia, pancreatitis and liver toxicity2. New therapies are needed to expand the current HAART repertoire, to provide hope for a cure and to reduce the cost of treatment an side effects 3.4

inhibitors ${ }^{9}$ and to look for synergistic anti-inflammatory compound pairs ${ }^{10}$, although the latter study did not yield any novel pairs. We have developed MuSIC, a pooled screening method, to screen a arge collection of diverse FDA-approved or clinically tested conpounds. The MuSIC screening library was designed to contain ten compounds in each well of a 384-well plate and cover all the possible pairwise combinations among these compounds using $<3 \%$ of the number of wells needed in a standard pairwise screen. For pools that contained potentially strong synergistic interactions, we deconvolute
each of them into 45 drug pairs in a secondary screen to identify eff -

Xu Tan et al., Nat Biotechnol. 2012, 30(11):1125-30 


\section{MuSIC screen}
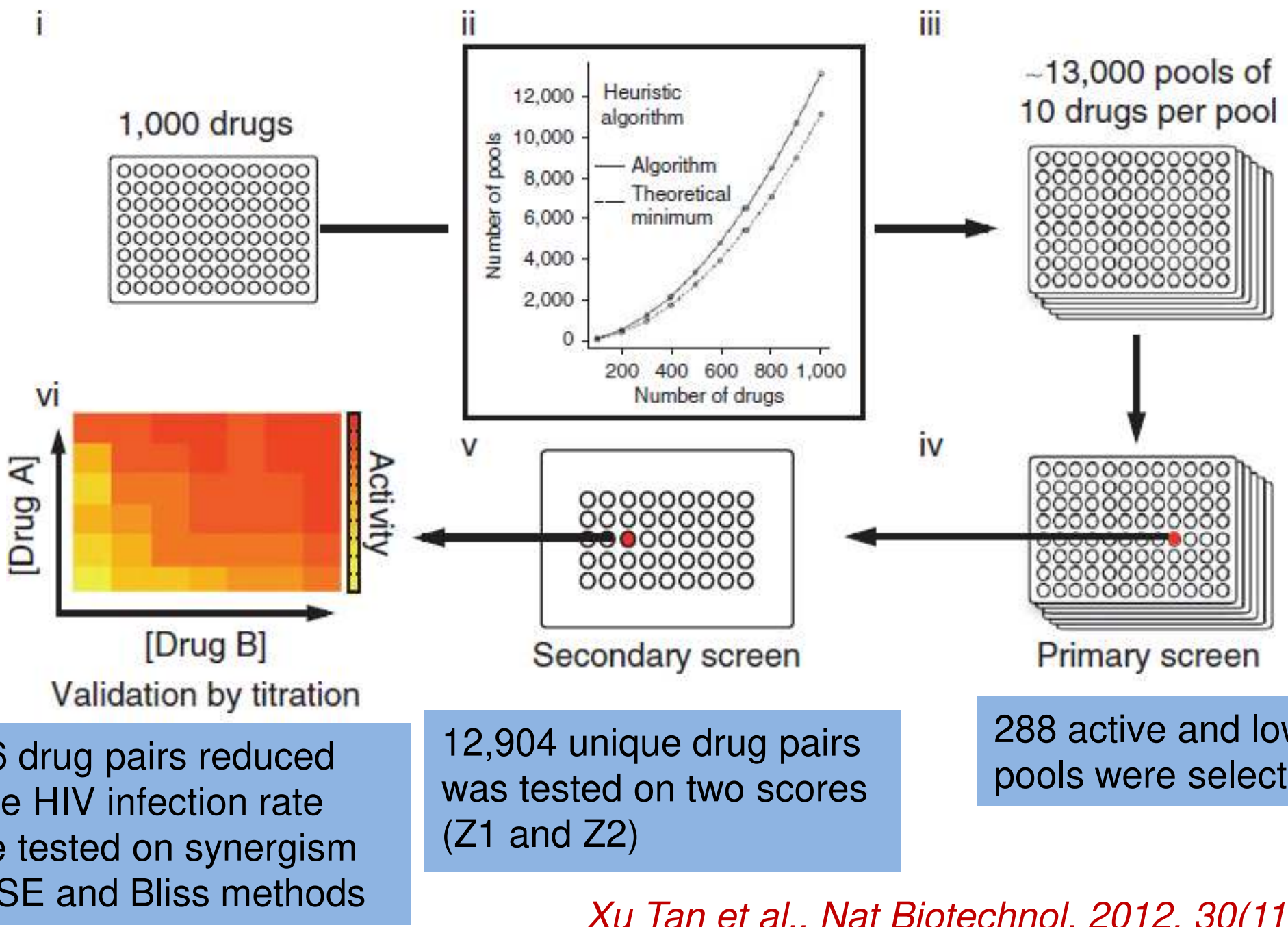

\section{6 drug pairs reduced the HIV infection rate were tested on synergism by HSE and Bliss methods}

12,904 unique drug pairs was tested on two scores (Z1 and Z2)

Xu Tan et al., Nat Biotechnol. 2012, 30(11):1125-30 


\section{GUSAR DDI}

- Self-Consistent Regression

- QNA (Quantitative Neighborhoods of Atoms) descriptors:

$P_{i}=B_{i} \sum_{k}(\operatorname{Exp}(-1 / 2 C))_{i k} B_{k} \quad Q_{i}=B_{i} \sum_{k}(\operatorname{Exp}(-1 / 2 C))_{i k} B_{k} A_{k}$

$A=1 / 2(I P+E A), B=(I P-E A)^{-1 / 2}$,

IP - ionization potential, EA - electron affinity

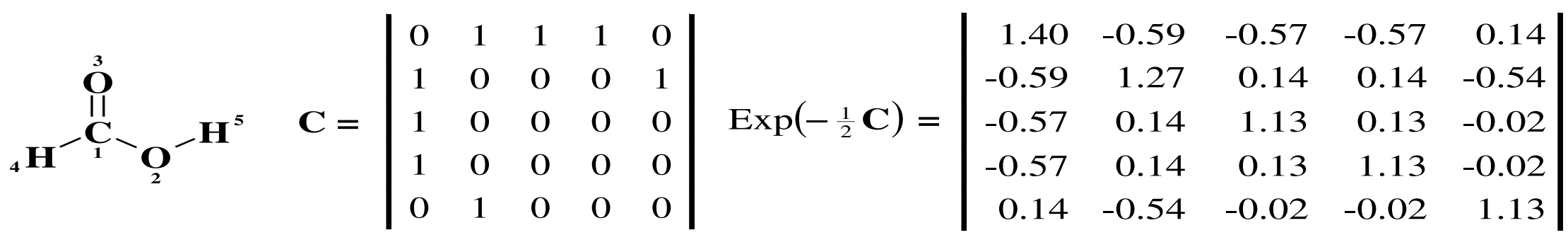

a)

b)

c) 


\section{QNA (Quantitative Neighborhoods of Atoms) descriptors:}

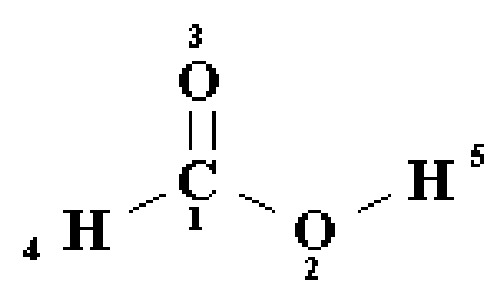

\begin{tabular}{ccccccc}
\hline & EA & IP & $A$ & $B$ & P & Q \\
\hline $\mathbf{C}$ & 1.263 & 11.26 & 6.262 & 0.316 & -0.00218 & -0.1820 \\
$\mathbf{O}$ & 1.461 & 13.62 & 7.541 & 0.287 & 0.02944 & 0.3019 \\
$\mathbf{O}$ & 1.461 & 13.62 & 7.541 & 0.287 & 0.06199 & 0.5297 \\
$\mathbf{H}$ & 0.754 & 13.60 & 7.177 & 0.279 & 0.05812 & 0.4706 \\
$\mathbf{H}$ & 0.754 & 13.60 & 7.177 & 0.279 & 0.05304 & 0.3533 \\
\hline
\end{tabular}

d)

Two-dimensional Chebyshev polynomials are used for approximating the functions $\mathrm{P}$ and $\mathrm{Q}$ over all atoms of the molecule.

\begin{tabular}{|l|l|l|}
\hline Structures & \\
\hline QNA descriptors & $\mathrm{P}^{1}, \mathrm{Q}^{1}:\left(\mathrm{P}_{1}^{1}, \mathrm{Q}_{1}^{1}\right),\left(\mathrm{P}_{2}^{1}, \mathrm{Q}_{2}^{1}\right), \ldots\left(\mathrm{P}_{n}^{1}, \mathrm{Q}_{n}^{1}\right)$ & $\mathrm{P}^{2}, \mathrm{Q}^{2}:\left(\mathrm{P}_{1}^{2}, \mathrm{Q}_{1}^{2}\right),\left(\mathrm{P}_{2}^{2}, \mathrm{Q}_{2}^{2}\right), \ldots\left(\mathrm{P}_{m}^{2}, \mathrm{Q}_{m}^{2}\right)$ \\
\hline $\begin{array}{l}\text { "Mixture" of QNA } \\
\text { descriptors }\end{array}$ & $\mathrm{P}^{1,2}, \mathrm{Q}^{1,2}:\left(\mathrm{P}_{1}^{1}, \mathrm{Q}_{1}^{1}\right),\left(\mathrm{P}_{2}^{1}, \mathrm{Q}_{2}^{1}\right), \ldots\left(\mathrm{P}_{n}^{1}, \mathrm{Q}_{n}^{1}\right),\left(\mathrm{P}_{1}^{2}, \mathrm{Q}_{1}^{2}\right),\left(\mathrm{P}_{2}^{2}, \mathrm{Q}_{2}^{2}\right), \ldots\left(\mathrm{P}_{m}^{2}, \mathrm{Q}_{m}^{2}\right)$ \\
\hline
\end{tabular}




\section{PASS DDI}

- Bayesian-like approach

- Pairs of Substances Multilevel Neighbourhoods of Atoms (PoSMNA) descriptors

PoSMNA is based on Multilevel Neighbourhoods of Atoms (MNA) descriptor:<smiles>O=C(O)c1ccccc1</smiles>

\section{MNA/1: $\quad \mathrm{C}(\mathrm{CN}-\mathrm{H})$}<smiles>O=C(O)c1ccccc1</smiles>

MNA/2: $\quad \mathrm{C}(\mathrm{C}(\mathrm{CC}-\mathrm{H}) \mathrm{N}(\mathrm{CC})-\mathrm{H}(\mathrm{C}))$

Filimonov D.A. et al. J. Chem. Inform. Computer Sci., 1999, 39, 666. Poroikov V.V. et al. J. Chem. Inform. Computer Sci., 2000, 40, 1349. 


\section{Pairs of Substances Multilevel Neighbourhoods of Atoms (PoSMNA) descriptors}

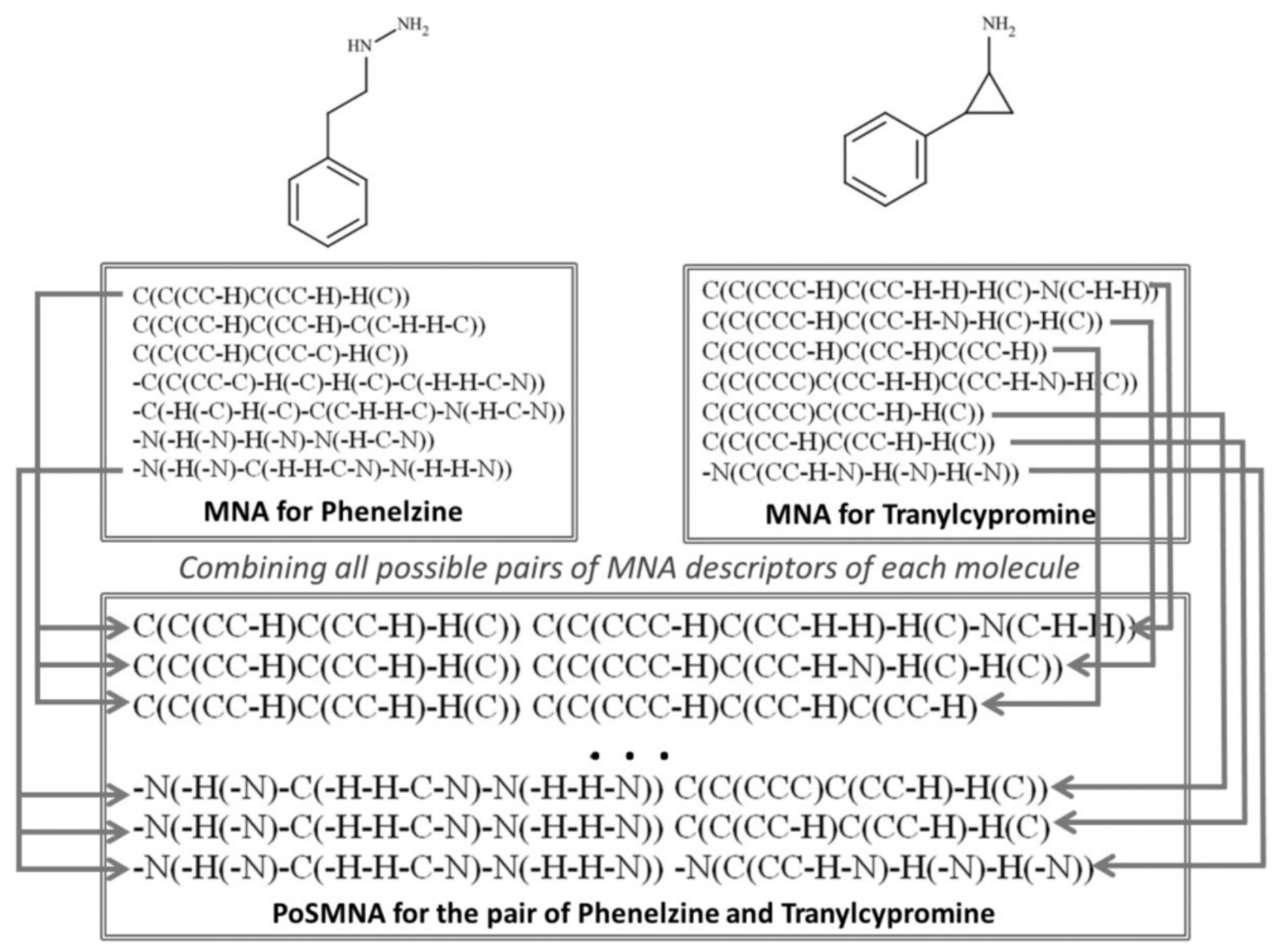

Dmitriev A. et al., Molecules 2019, 24(21), 3955 


\section{Accuracy of SAR models created on 116 drug pairs results of synergistic studies}

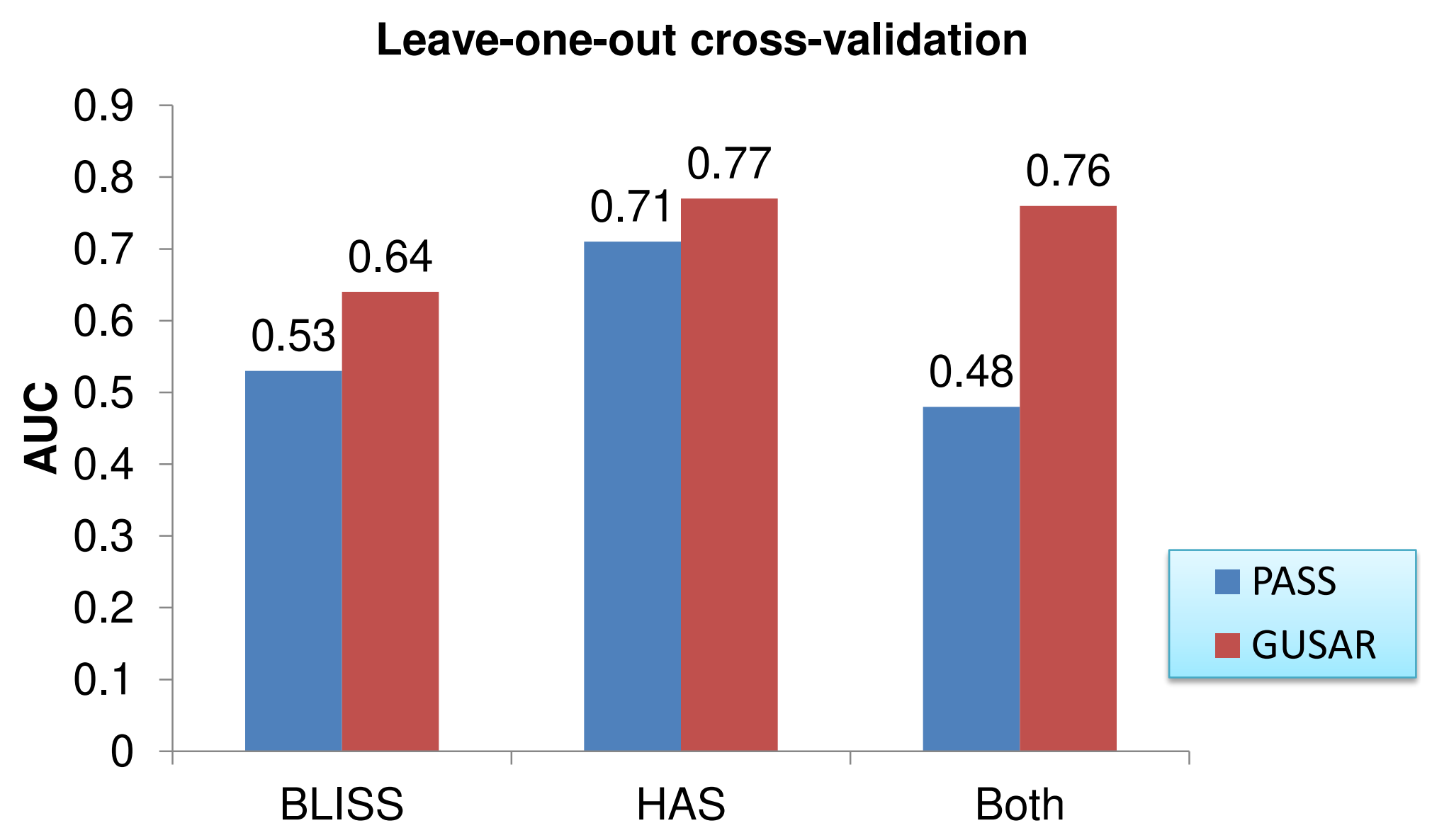




\section{Can we select candidate synergistic drug pairs based on $\mathrm{Z} 1$ and $\mathrm{Z2}$ scores?}

116 drug pairs were selected based on the highest values of $Z 1$ and $Z 2$ scores

\begin{tabular}{|c|c|c|}
\hline \multicolumn{3}{|c|}{ Number of drug pairs. Experimental data } \\
\hline Method & Synergy & No synergy \\
\hline BLISS & 66 & 50 \\
\hline HSA & 77 & 39 \\
\hline Both & 41 & 75 \\
\hline
\end{tabular}

Yes! From 35\% (Both) to 66\% (HSA) selected drug pairs revealed anti-HIV synergism measured by different methods.

Xu Tan et al., Nat Biotechnol. 2012, 30(11):1125-30 


\section{Accuracy of SAR models created based on Z score data}

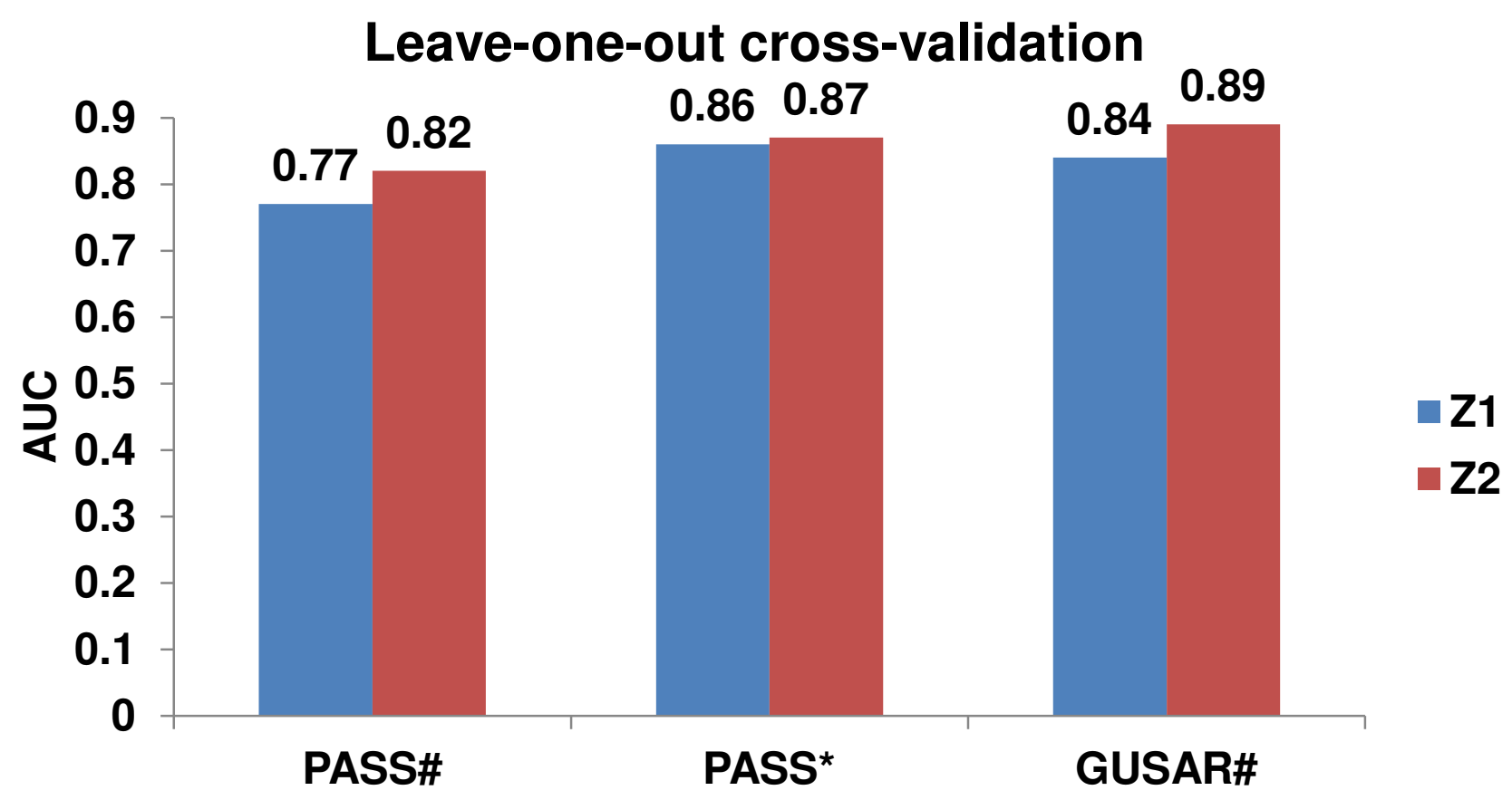

\# - the structures for 12,000 drug pairs with Z1 or Z2 scores were used as the training set

- - the structures for $~ 12,000$ drug pairs with Z1 or Z2 scores + all drug pairs $(\sim 150,000)$ from the first pool, which showed no anti-HIV activity.

Drug pairs with Z1 or Z2 scores with less -1 values were considered as actives, others were considered as inactives. 


\section{Accuracy of synergism prediction for 116 drug pairs based on the results of SAR models created using $Z$ score data}

\begin{tabular}{|c|c|c|c|c|c|c|}
\hline & PASS DDI & & \multicolumn{3}{|c|}{ GUSAR DDI } & \multirow[b]{2}{*}{ Acc } \\
\hline Methods & Sen & Spec & Acc & Sen & Spec & \\
\hline \multicolumn{7}{|c|}{ Z score 12000 part 1} \\
\hline BLISS & 0.85 & 0.29 & 0.61 & 0.85 & 0.20 & 0.57 \\
\hline HSA & 0.73 & 0.10 & 0.52 & 0.76 & 0.05 & 0.52 \\
\hline Both & 0.80 & 0.23 & 0.42 & 0.78 & 0.15 & 0.37 \\
\hline \multicolumn{7}{|c|}{ Z score 12000 part 2} \\
\hline BLISS & 0.85 & 0.29 & 0.61 & 0.80 & 0.33 & 0.60 \\
\hline HSA & 0.71 & 0.05 & 0.48 & 0.65 & 0.08 & 0.46 \\
\hline Both & 0.77 & 0.20 & 0.40 & 0.70 & 0.23 & 0.39 \\
\hline \multicolumn{7}{|c|}{ Z score 12000 part 1 and Z score 12000 part 2} \\
\hline BLISS & 0.83 & 0.33 & 0.61 & 0.77 & 0.35 & 0.59 \\
\hline HSA & 0.69 & 0.10 & 0.49 & 0.61 & 0.08 & 0.43 \\
\hline Both & 0.78 & 0.24 & 0.43 & 0.65 & 0.24 & 0.39 \\
\hline \multicolumn{7}{|c|}{$Z$ score part $1+$ all inactive } \\
\hline BLISS & 0.89 & 0.20 & 0.60 & & & \\
\hline HSA & 0.81 & 0.08 & 0.56 & & & \\
\hline Both & 0.88 & 0.16 & 0.41 & & & \\
\hline \multicolumn{7}{|c|}{$Z$ score part $2+$ all inactive } \\
\hline BLISS & 0.84 & 0.29 & 0.60 & & & \\
\hline HSA & 0.72 & 0.05 & 0.49 & & & \\
\hline Both & 0.80 & 0.20 & 0.41 & & & \\
\hline \multicolumn{7}{|c|}{$Z$ score part $1+$ all inactive and $Z$ score part $2+$ all inactive } \\
\hline BLISS & 0.85 & 0.33 & 0.62 & & & \\
\hline HSA & 0.71 & 0.10 & 0.50 & & & \\
\hline Both & 0.80 & 0.24 & 0.44 & & & \\
\hline
\end{tabular}




\section{Selection of new possible synergistic anti-HIV drug pair combinations}

- 997 FDA approved drugs different from 1000 drugs used in MuSIC screen were selected from DrugBank 4.1.

- They were used for generation of the set of $\sim 500,000$ new drug pairs.

- The set was used for two step virtual screening based on PASS DDI (the results given on the training sets with $Z$ scores (Z score part $1+$ all inactive and $Z$ score part $2+$ all inactive data) and GUSAR DDI (the training set with 116 drug pairs (Both data)) models. 


\section{PASS DDI prediction results for $\sim 500,000$ drug pairs}

\begin{tabular}{|l|r|rrr|} 
& Pa>Pi & Pa>0.3 & Pa>0.5 & $P a>0.7$ \\
\hline Z1 score & 119244 & 69362 & 32080 & 6606 \\
\hline Z2 score & 112869 & 51841 & 14532 & 5635 \\
\hline
\end{tabular}

$\mathrm{Pa}-\mathrm{a}$ probability that a pair of drugs displays anti-HIV activity $\mathrm{Pi}$ - a probability that a pair of drugs does not display anti-HIV activity

Intersection of Z1 and Z2 with Pa>0.7 = 5430 drug pairs 


\section{GUSAR DDI prediction results}

- 1974 new drug pairs were predicted by GUSAR DDI to be anti-HIV synergistic from 5430 drug pairs selected based on PASS DDI prediction results for Z1 and Z2 scores.

- 89 new drug pairs were predicted to be anti-HIV synergistic with probability $>60 \%$

- 5 new drug pairs were predicted to be anti-HIV synergistic with probability $>70 \%$ :

-Auranofin/Fluocinonide

-Auranofin/Difluprednate

-Auranofin/Fluocinolone Acetonide

-Bismuth Subsalicylate/Fluocinonide

-Icosapent/Difluprednate 


\section{Targets of the selected drug pairs (DrugBank data)}

\begin{tabular}{|c|c|c|c|}
\hline Drug & Target & Drug & Target \\
\hline \multirow[t]{9}{*}{ Auranofin } & Peroxiredoxin-5, mitochondrial & Fluocinonide & Glucocorticoid receptor \\
\hline & Inhibitor of nuclear factor kappa-B kinase subunit beta & & Smoothened homolog \\
\hline & & Difluprednate & Glucocorticoid receptor \\
\hline & & $\begin{array}{l}\text { Fluocinolone } \\
\text { Acetonide }\end{array}$ & Glucocorticoid receptor \\
\hline & & & Annexin A1 \\
\hline & & & Annexin A2 \\
\hline & & & Annexin A3 \\
\hline & & & Annexin A4 \\
\hline & & & Annexin A5 \\
\hline \multirow[t]{2}{*}{$\begin{array}{l}\text { Bismuth } \\
\text { Subsalicylate }\end{array}$} & Serotransferrin & Fluocinonide & Glucocorticoid receptor \\
\hline & & & Smoothened homolog \\
\hline \multirow[t]{12}{*}{ Icosapent } & Prostaglandin G/H synthase 2 & Difluprednate & Glucocorticoid receptor \\
\hline & Prostaglandin $\mathrm{G} / \mathrm{H}$ synthase 2 & & \\
\hline & AProstaglandin $\mathrm{G} / \mathrm{H}$ synthase 1 & & \\
\hline & APeroxisome proliferator-activated receptor gamma & & \\
\hline & APeroxisome proliferator-activated receptor delta & & \\
\hline & UFatty acid desaturase 1 & & \\
\hline & ULong-chain-fatty-acid--CoA ligase 4 & & \\
\hline & ULong-chain-fatty-acid--CoA ligase 3 & & \\
\hline & AFree fatty acid receptor 1 & & \\
\hline & ASodium/calcium exchanger 1 & & \\
\hline & $\begin{array}{l}\text { UTransient receptor potential cation channel subfamily } \mathrm{V} \\
\text { member } 1\end{array}$ & & \\
\hline & UPeroxisome proliferator-activated receptor alpha & & \\
\hline
\end{tabular}




\section{Comparison with the experimental data given by MuSIC screen}

Betamethazone $+\mathrm{NTZ}$ - the most active drug pair

- Betamethasone - Glucocorticoid receptor (target)

- NTZ (Nitazoxanide) - Pyruvate-flavodoxin oxidoreductase (catalyzes the ferredoxin-dependent oxidative decarboxylation of pyruvate). It has been also studied as a broad-spectrum antiviral agent due to its ability to inhibit the replication of several RNA and DNA viruses.

b

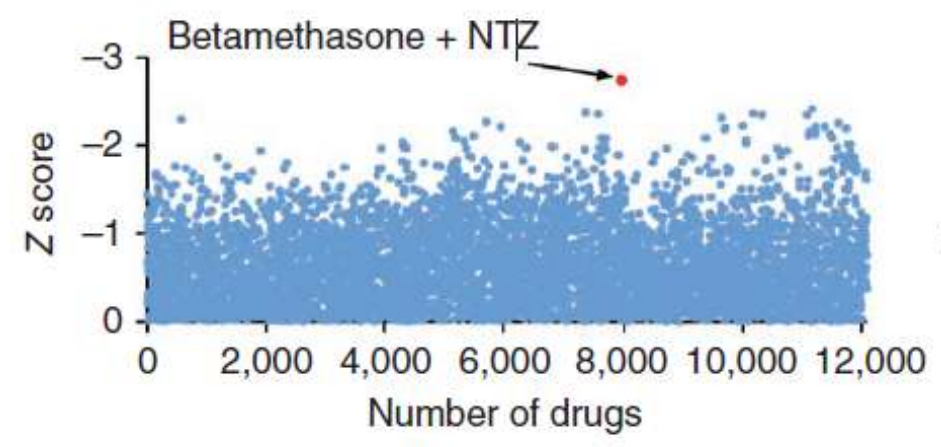

C

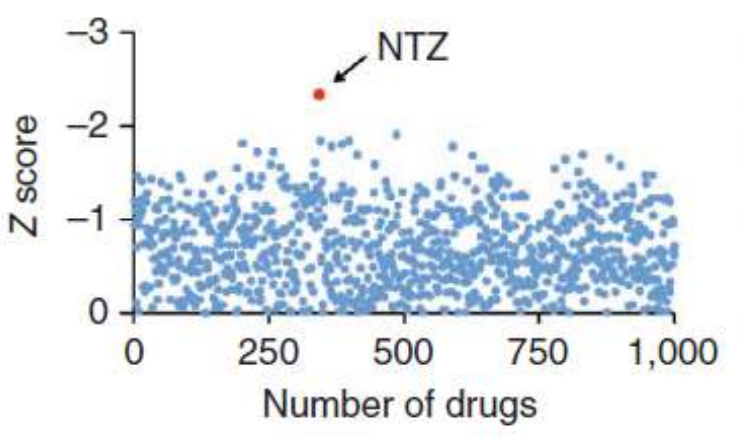

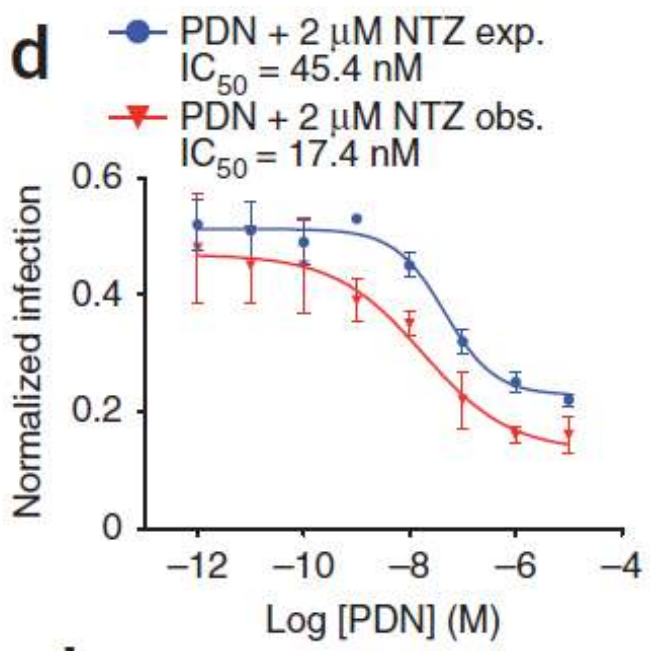

(M)

Xu Tan et al., Nat Biotechnol. 2012, 30(11):1125-30 


\section{Acknowledgments}

This work was supported by Russian Science Foundation grant [number 19-75-10097].

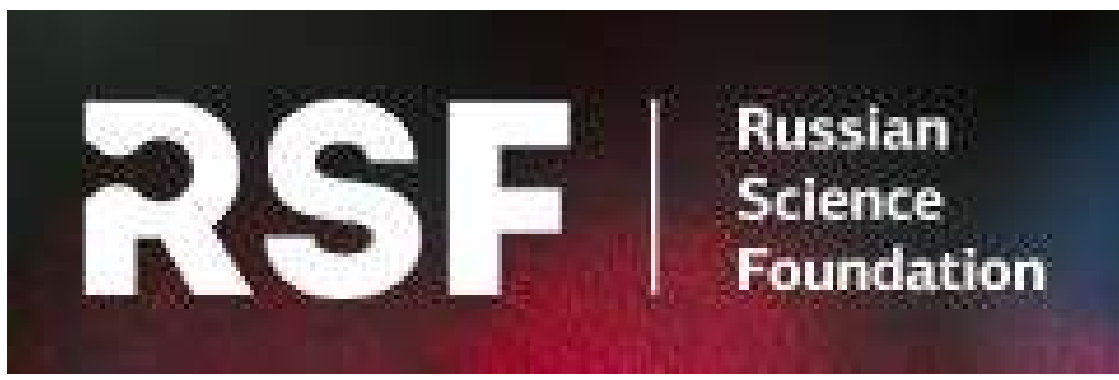

